\title{
Using a quality improvement model to enhance providers' performance in maternal and newborn health care: a post- only intervention and comparison design
}

Firew Ayalew ${ }^{1 *}$, Gizachew Eyassu', Negash Seyoum¹, Jos van Roosmalen², Eva Bazant ${ }^{3}$, Young Mi Kim³, Alemnesh Tekleberhan' ${ }^{1}$, Hannah Gibson ${ }^{1}$, Ephrem Daniel ${ }^{1}$ and Jelle Stekelenburg ${ }^{4,5}$

\begin{abstract}
Background: The Standards Based Management and Recognition (SBM- ${ }^{\top}$ ) approach to quality improvement has been implemented in Ethiopia to strengthen routine maternal and newborn health (MNH) services. This evaluation assessed the effect of the intervention on MNH providers' performance of routine antenatal care (ANC), uncomplicated labor and delivery and immediate postnatal care (PNC) services.

Methods: A post-only evaluation design was conducted at three hospitals and eight health centers implementing SBM-R and the same number of comparison health facilities. Structured checklists were used to observe MNH providers' performance on ANC (236 provider-client interactions), uncomplicated labor and delivery (226 provider-client interactions), and immediate PNC services in the six hours after delivery (232 provider-client interactions); observations were divided equally between intervention and comparison groups. Main outcomes were provider performance scores, calculated as the percentage of essential tasks in each service area completed by providers. Multilevel analysis was used to calculate adjusted mean percentage performance scores and standard errors to compare intervention and comparison groups.

Results: There was no statistically significant difference between intervention and comparison facilities in overall mean performance scores for ANC services (63.4\% at intervention facilities versus $61.0 \%$ at comparison facilities, $p=0.650$ ) or in any specific ANC skill area. MNH providers' overall mean performance score for uncomplicated labor and delivery care was 11.9 percentage points higher in the intervention than in the comparison group $(77.5 \%$ versus $65.6 \% ; p=0.002)$. Overall mean performance scores for immediate PNC were 22.2 percentage points higher at intervention than at comparison facilities (72.8\% versus 50.6\%; $p=0.001)$; and there was a significant difference of 22 percentage points between intervention and comparison facilities for each PNC skill area: care for the newborn and health check for the mother.

Conclusions: The SBM-R quality improvement intervention made a significant positive impact on MNH providers' performance during labor and delivery and immediate PNC services, but not during ANC services. Scaling up the intervention to other facilities and regions may increase the availability of good quality MNH services across Ethiopia. The findings will also guide implementation of the government's five-year (2015-2020) health sector transformation plan and health care quality strategies needed to meet the country's MNH goals.
\end{abstract}

Keywords: SBM-R, Ethiopia, Antenatal care, Postnatal care, Labor and delivery, Provider performance, Quality improvement, Maternal health, Newborn health

* Correspondence: Firew.Ayalew@Jhpiego.org

'Jhpiego Ethiopia, Addis Ababa, Ethiopia

Full list of author information is available at the end of the article 


\section{Background}

Maternal and child mortality have declined over the past 20 years in Ethiopia. However, 2015 estimates show that mortality remains high in the months before and after childbirth, with 420 mothers dying per 100,000 live births, 41 infants dying per 1000 live births, 28 neonates dying per 1000 live births and neonatal deaths accounting for $47 \%$ of all under-five mortality [1]. In line with Sustainable Development Goals, by 2020 Ethiopia aims to reduce the maternal mortality ratio to 199 per 100,000 live births, the infant mortality rate to 20 per 1000 live births and the neonatal mortality rate to 10 per 1000 live births [2].

Achieving these goals will require improvements in the quality of maternal and newborn care services. The World Health Organization (WHO) defines eight standards for improving the quality of maternal and newborn care, one of which calls for "competent, motivated staff to be consistently available to provide routine care and manage complications" [3]. Multiple factors influence health workers' performance, including health workers' knowledge and skills gaps, caseload, patient demand, clinical practices, educational opportunities, availability of supplies and equipment and also the presence of a quality improvement process [4].

Various quality improvement models have been implemented in low, middle and high-income countries to improve healthcare providers' performance and quality of services [5, 6]. WHO has concluded that any of these strategies can help to ensure high quality antenatal, intrapartum, and postnatal care [3].

Multifaceted interventions that include supervision, training, audit and feedback have been shown to have a greater impact than single interventions on the quality of health workers' performance [5, 7]. One such approach is Standards-Based Management and Recognition $\left(\mathrm{SBM}-\mathrm{R}^{\odot}\right)$, which includes four stages to improve performance and quality of health services [8].

In Ethiopia, the Ministry of Health $(\mathrm{MOH})$ began implementing the SBM-R quality improvement model in 2003 to improve HIV/AIDS and maternal and newborn health $(\mathrm{MNH})$ services. However, the effect of SBM-R on healthcare providers' performance and the quality of MNH services has not been evaluated in Ethiopia. This study seeks to address gaps in the literature regarding the effectiveness of MNH quality improvement interventions $[3,9]$ on providers' performance by evaluating the impact of the SBM-R intervention in Ethiopia.

The evaluation asked: Do MNH providers in facilities that have implemented SBM-R perform better than providers at comparison facilities during routine delivery of antenatal care (ANC), uncomplicated labor and delivery, and immediate postnatal care (PNC) services within the first six hours after delivery [10]?

\section{Methods}

\section{Study design and setting}

This study employed a post-only intervention and comparison evaluation design to assess the impact of the SBM-R intervention on provider performance at public health facilities located in four major regions of Ethiopia: Tigray, Amhara, Oromia and Southern Nations, Nationalities and Peoples' (SNNP). An SBM-R process to improve the performance and quality of $\mathrm{MNH}$ services was introduced at 116 government health facilities (15 hospitals and 101 health centers) from March 2011 to June 2014 with a phased-in approach. This study focuses on the 21 health facilities (15 health centers and 6 hospitals) in the first phase. All of these facilities had proceeded through the entire SBM-R process within three years of implementation and were ready for external assessment.

\section{Description of SBM-R intervention}

Guided by national $\mathrm{MNH}$ policies and guidelines, performance standards in 10 technical areas were developed for providing quality $\mathrm{MNH}$ services, including 80 standards for health centers and 79 standards for hospitals. Technical areas included: routine ANC, uncomplicated labor and delivery care and post-partum care; management of antenatal, intrapartum, and postpartum complications; infection prevention; laboratory; pharmacy; human, physical and materials resources; information, education, communication and community participation; and management system. Each standard comprised a series of specific items that could be scored as a measure of achievement. After authorities endorsed the standards, they were introduced in each health facility. Health managers and providers received three rounds of SBM-R modular trainings focusing on how to use and implement the standards, measure progress, and recognize performance.

After completing the first round of SBM-R training, each facility established a quality improvement team to implement the intervention. This team conducted a baseline assessment of services at the facility using standard tools to identify performance gaps and analyze root causes. Commonly identified performance gaps included deficits in health providers' skills and knowledge as well as shortages of supplies and equipment. Based on the initial gap assessment, intervention facilities received essential equipment and supplies (such as newborn weighing scales, autoclaves, examination beds, delivery beds, baby towels and hats, and infection prevention supplies), training on Basic Emergency Obstetric Newborn Care (BEmONC) for providers, and regular follow-up from regional health bureaus and partners, including site mentoring, review meetings and quarterly supportive supervision. Quality improvement teams at each facility conducted assessments every six 
months to track performance using direct structured observations, document review and provider interviews. Based on the results, the teams developed action plans to address remaining performance gaps. All target facilities completed the four stages of SBM-R and were eligible for external assessments verifying that they had achieved at least $80 \%$ of the standards.

\section{Sampling and sample selection procedure}

Sample sizes were calculated for observations of providers' performance in each of three service areas (ANC, labor and delivery, and immediate PNC) based on 95\% level of statistical confidence, $80 \%$ statistical power, $20 \%$ expected difference in health providers' performance between intervention and comparison groups and the recommended value of $1 \%$ intraclass correlation coefficient for median value of primary health care research [11]. The intraclass correlation coefficient is the correlation between responses given by randomly selected respondents within clusters or facilities to measure degree of similarity [12].

Health providers' mean performance scores from a 2010 quality of care study in Ethiopia [13] informed the reference values used to calculate the three sample sizes. For ANC, a mean percentage performance score of $38 \%$ for preventive treatments, including the administration of tetanus toxoid and prescription or provision of iron/ folic acid was used. For uncomplicated labor and delivery care, a mean percentage performance score of $29 \%$ on active management of the third stage of labor was used. For immediate PNC, a mean percentage performance score of $47 \%$ on immediate newborn care services was used. Based on these, we calculated sample sizes of 236 provider-client interactions (observations) for ANC, 226 for uncomplicated labor and delivery care, and 232 for immediate PNC. Each sample was divided equally between intervention and comparison groups.

A total of six hospitals and 15 health centers had completed all stages of SBM-R. Half (three hospitals and eight health centers) were randomly selected for the study sample. Comparison facilities that had not implemented SBM-R (three hospitals and eight health centers) were selected from the same region and zone as the intervention facilities, but from different districts. Comparison facilities were matched with intervention facilities on staff size and client volume, including the number of first ANC visits and deliveries attended by skilled birth attendants.

The estimated number of $\mathrm{MNH}$ providers (those who offer ANC, labor and delivery, and/or PNC services) at each facility was 12 per hospital and five per health center, for a total of 152 across all intervention and comparison facilities in the sample. Because the number of
MNH providers at each facility was small, all of them were invited to participate in the study. Women who sought ANC or labor and delivery followed by immediate PNC at these facilities during the study period were invited to participate.

\section{Observation tool}

Observations of service delivery using structured checklists were conducted to measure provider performance in ANC, uncomplicated labor and delivery care, and immediate PNC. The clinical observation tool listed essential maternity and newborn care tasks and was adapted from an earlier quality of care study in Ethiopia [13], national BEmONC training guidelines [10], and SBM-R tools. The ANC portion of the tool consisted of 53 tasks in eight skill areas; the labor and delivery portion consisted of 105 tasks in 10 skill areas; and the PNC portion included nine tasks in two skill areas. Observers coded each task as "Yes, task performed" or "No, task not performed." The tool also captured basic characteristics of health providers.

\section{Data collection}

Data were collected from July 7 to August 3, 2014 by 20 experienced assessors. Assessors consisted of midwives and health officers with at least a Bachelor of Science degree and national BEmONC master trainers. The assessors attended a four-day training on consent, study instruments, observation techniques, and data quality, and demonstrated their competency through role plays on consents and observation checklists before deployment. A team of two assessors conducted observations at each facility. Five supervisors and one study coordinator supervised them. To minimize observer's bias, assessors were not assigned to health facilities where they worked.

Assessors collected information on providers' sociodemographic characteristics and conducted observations while providers performed each step of routine ANC services, all stages of uncomplicated labor and delivery care, and/or the full range of immediate PNC services within the first six hours after delivery. Assessors stayed overnight at facilities to conduct observations, remaining up to five days at each facility until they observed at least one provider-client interaction per facility. Providers could be observed with more than one patient during this time. If no delivery took place in the facility during the assessors' visit, the study coordinator instructed assessors to move to the next sample facility and observe more providers to compensate. Before traveling to the next sample facility, assessors and supervisors checked the completed observation tools for completeness and consistency. 


\section{Data management and analysis}

Data was cleaned and entered into CSPro V 5.0 and exported to STATA V 13.1 for statistical analysis. Descriptive statistics, including frequency and percentages, were computed to identify outliers and missing values. Data were nested in three levels: clients (first level) were clustered by provider (second level), and providers were clustered by facility (third level); this nesting structure creates dependency of data. The analysis examined three outcome variables: provider performance scores on ANC, uncomplicated labor and delivery care, and immediate PNC, calculated as the percentage of essential tasks in each service area completed by providers. For this study, health providers' performance is defined as the extent to which actual work practices reflect the skills and knowledge taught in training using standardized operating procedures [14].

Multilevel analysis was used to calculate adjusted mean percentage performance scores and standard errors to compare intervention and comparison groups. The multilevel model is appropriate to generate adjusted standard errors of the estimates and test statistics or $p$-values for clustered data, instead of conventional linear models that overestimate test statistics and lead to misleading inferences $[15,16]$. Mean performance scores ranged from 0 to 1 . The mean percentage performance score was calculated by dividing mean performance scores by the maximum mean score and multiplying by 100 .

Interclass correlation coefficients (ICC) were computed to assess variations explained by facility and health provider clustering effects on three outcome variables: health provider performance on ANC, uncomplicated labor and delivery care, and immediate PNC. ICC ranges from 0 to 1 and is used to estimate the degree of providers' performance similarity within and among clusters, where an ICC value close to 1 indicates similarities between individual's performance within clusters and an ICC value close to 0 shows independence of individual's performance within clusters and/or an indication to use single level analysis [17]. ICC values suggest the use of multilevel analysis even if they are close to 0.05 [18].

A Chi-square test was used to assess associations of study participants and facility characteristics among intervention and comparison groups. A $p$-value of less than 0.05 was used for all statistical tests. We computed adjusted standard errors of the mean performance scores to calculate $95 \%$ of confidence interval of the mean.

\section{Ethical considerations}

Study approvals were obtained from the Ethiopian Ministry of Science and Technology National Research Ethics Review Committee and the Johns Hopkins Bloomberg School of Public Health Institutional Review
Board. Trained assessors informed health providers and women sought MNH services about the study and asked for their written consent to be observed. Woman was asked to allow trained assessor to observe only her labor and delivery services with the provider however, assessors did not collect data on her name, identification number and pregnancy history. For those women who arrived to the facility with labor pain, her next of kin was asked for written consent for observation. If a woman had complication, assessors did not ask written consent from a woman or her next of kin; and they waited another women who came for labor and delivery services to use for providers observations.

\section{Results}

\section{Sample characteristics}

The sample consisted of $147 \mathrm{MNH}$ providers (79 in the SBM-R intervention group and 68 in the comparison group). Most providers were female in both intervention (77.2\%) and comparison (70.6\%) groups, and one-third were over age 30 (Table 1). A large majority were midwives and nurses, and about half worked at hospitals and half at health centers. Almost half had fewer than five years of work experience in both intervention (48.1\%) and comparison (45.6\%) groups. There were no significant differences in provider or facility characteristics between intervention and comparison groups.

\section{Degree of similarity of MNH providers' performance at facility and provider levels}

As shown in Table 2, assessors observed 232 ANC clients who were attended by 63 different health providers, 226 labor and delivery clients who were observed with 117 different providers and 229 PNC clients who were observed with 109 providers. ICC values vary, but are higher in the intervention than in the comparison group with one exception (PNC services at the facility level). This suggests that the quality of services offered was more uniform at intervention than comparison facilities. The greatest differences between intervention and comparison groups were seen in labor and delivery services: provider performance was more similar within each facility in intervention $(\mathrm{ICC}=0.775)$ than in comparison facilities $(\mathrm{ICC}=0.454)$, and performance was also more similar for multiple observations of the same provider in intervention $(\mathrm{ICC}=0.632$ ) than in comparison facilities $(\mathrm{ICC}=0.474)$.

\section{Health provider performance ANC services}

Average overall performance scores for ANC services were $63.4 \%$ in the intervention and $61.0 \%$ in the comparison group $(p=0.650)$. There were no significant differences between study groups in any of the eight skill 
Table 1 Characteristics of providers observed

\begin{tabular}{|c|c|c|c|c|c|}
\hline \multirow{2}{*}{$\begin{array}{l}\text { Demographic and work- } \\
\text { related characteristics }\end{array}$} & \multicolumn{2}{|c|}{ Intervention group $(n=79)$} & \multicolumn{2}{|c|}{ Comparison group $(n=68)$} & \multirow{2}{*}{$\begin{array}{l}p \text {-value (Chi } \\
\text { square test) }\end{array}$} \\
\hline & n & $\%$ & $\bar{n}$ & $\%$ & \\
\hline \multicolumn{6}{|l|}{ Gender } \\
\hline Female & 61 & 77.2 & 48 & 70.6 & \multirow[t]{2}{*}{0.360} \\
\hline Male & 18 & 22.8 & 20 & 29.4 & \\
\hline \multicolumn{6}{|l|}{ Age } \\
\hline$<25$ years & 23 & 29.1 & 27 & 39.7 & \multirow[t]{3}{*}{0.337} \\
\hline $25-30$ years & 28 & 35.5 & 18 & 26.5 & \\
\hline Over 30 years & 28 & 35.4 & 23 & 33.8 & \\
\hline \multicolumn{6}{|l|}{ Qualification } \\
\hline Nurse & 29 & 36.7 & 28 & 41.2 & \multirow[t]{3}{*}{0.746} \\
\hline Midwife & 41 & 51.9 & 31 & 45.6 & \\
\hline Other (doctor, health officer) & 9 & 11.4 & 9 & 13.2 & \\
\hline \multicolumn{6}{|c|}{ Years of work experience in the health care system (private and public) } \\
\hline$<5$ years & 38 & 48.1 & 31 & 45.6 & \multirow[t]{3}{*}{0.856} \\
\hline 5 to 10 years & 20 & 25.3 & 20 & 29.4 & \\
\hline$>10$ years & 21 & 26.6 & 17 & 25.0 & \\
\hline \multicolumn{6}{|l|}{ Type of facility } \\
\hline Hospital & 40 & 50.6 & 36 & 52.9 & \multirow[t]{2}{*}{0.780} \\
\hline Health center & 39 & 49.4 & 32 & 47.1 & \\
\hline \multicolumn{6}{|l|}{ Region } \\
\hline Amhara & 16 & 20.3 & 15 & 22.1 & \multirow[t]{4}{*}{0.529} \\
\hline Oromia & 25 & 31.7 & 24 & 35.3 & \\
\hline SNNP & 22 & 27.8 & 12 & 17.6 & \\
\hline Tigray & 16 & 20.2 & 17 & 25.0 & \\
\hline
\end{tabular}

areas, although performance scores were higher by over 10 percentage points for rapid initial assessment, preventive treatment and counseling on danger signs in intervention as compared to comparison facilities. Health providers' strengths and weaknesses, as evidenced by higher and lower scores in the various skill areas, were similar in both study groups (Table 3 ).

\section{Uncomplicated labor and delivery services}

Average overall performance scores for uncomplicated labor and delivery services were significantly higher in the intervention than in the comparison group $(77.5 \%$ versus $65.6 \%, p=0.002)$. $\mathrm{MNH}$ providers at intervention facilities significantly outperformed those at comparison facilities in 7 of 10 skill areas. Differences between the study groups were greatest for rapid initial assessment $(60.6 \%$ versus $42.8 \%, p=0.019)$, care during labor $(81.1 \%$ versus $66.0 \%, p=0.001$ ), and immediate newborn care (76.9\% versus $61.9 \%, p=0.013$ ). Differences between the study groups were smallest (less than 10 percentage points) and not significant for quality utilization of partograph and infection prevention (Table 3).

Table 2 Number of MNH providers and provider-client interactions observed, and intraclass correlation coefficients

\begin{tabular}{|c|c|c|c|c|c|c|c|c|c|}
\hline \multirow[t]{2}{*}{ Service area } & \multicolumn{3}{|c|}{ Number of MNH providers observed } & \multicolumn{3}{|c|}{ Number of provider-client interactions observed } & \multicolumn{3}{|c|}{ Intraclass Correlation Coefficient (ICC) } \\
\hline & Total & $\begin{array}{l}\text { Inter-vention } \\
\text { group }\end{array}$ & $\begin{array}{l}\text { Com-parison } \\
\text { group }\end{array}$ & Total & $\begin{array}{l}\text { Inter-vention } \\
\text { group }\end{array}$ & $\begin{array}{l}\text { Com-parison } \\
\text { group }\end{array}$ & Cluster level & $\begin{array}{l}\text { Inter-vention } \\
\text { group }\end{array}$ & $\begin{array}{l}\text { Com-parison } \\
\text { group }\end{array}$ \\
\hline \multirow[t]{2}{*}{ ANC } & 63 & 29 & 34 & 232 & 118 & 114 & Facility & 0.551 & 0.458 \\
\hline & & & & & & & Provider & 0.763 & 0.667 \\
\hline \multirow[t]{2}{*}{ Labor \& delivery } & 117 & 57 & 60 & 226 & 123 & 123 & Facility & 0.632 & 0.474 \\
\hline & & & & & & & Provider & 0.775 & 0.454 \\
\hline \multirow[t]{2}{*}{ PNC } & 109 & 57 & 52 & 229 & 119 & 110 & Facility & 0.530 & 0.573 \\
\hline & & & & & & & Provider & 0.658 & 0.574 \\
\hline
\end{tabular}


Table 3 Adjusted mean provider performance scores, by service area and study group

\begin{tabular}{|c|c|c|c|c|c|c|c|}
\hline \multirow[t]{2}{*}{ Skill areas } & \multirow{2}{*}{$\begin{array}{l}\text { Number of } \\
\text { items }\end{array}$} & \multicolumn{2}{|c|}{ Intervention group } & \multicolumn{2}{|c|}{ Comparison group } & \multirow{2}{*}{$\begin{array}{l}\text { \% point difference } \\
\text { between study groups }\end{array}$} & \multirow[t]{2}{*}{$p$-value ${ }^{a}$} \\
\hline & & $\begin{array}{l}\text { Mean } \\
\text { score }\end{array}$ & $\begin{array}{l}\text { Standard } \\
\text { error }\end{array}$ & $\begin{array}{l}\text { Mean } \\
\text { score }\end{array}$ & $\begin{array}{l}\text { Standard } \\
\text { error }\end{array}$ & & \\
\hline \multicolumn{8}{|c|}{ Antenatal care ( $n=124$ for intervention group; $n=120$ for comparison group) } \\
\hline Rapid initial assessment & 6 & 56.5 & 0.07 & 40.8 & 0.07 & +15.7 & 0.099 \\
\hline Preventive treatment & 3 & 58.8 & 0.05 & 47.7 & 0.06 & +11.1 & 0.140 \\
\hline Counseling on danger signs & 6 & 69.7 & 0.06 & 59.1 & 0.05 & +10.6 & 0.174 \\
\hline Friendly reception for women & 2 & 58.6 & 0.07 & 50.0 & 0.07 & +8.6 & 0.346 \\
\hline Obstetrical history taking & 11 & 81.8 & 0.07 & 77.4 & 0.07 & +4.4 & 0.651 \\
\hline Laboratory screening tests & 3 & 82.6 & 0.10 & 79.0 & 0.10 & +3.6 & 0.783 \\
\hline Physical examination & 11 & 73.8 & 0.03 & 70.4 & 0.03 & +3.4 & 0.380 \\
\hline Birth preparedness & 11 & 45.2 & 0.07 & 43.5 & 0.07 & +1.7 & 0.858 \\
\hline Overall (composite) score & 53 & 63.4 & 0.04 & 61.0 & 0.04 & +2.4 & 0.650 \\
\hline \multicolumn{8}{|c|}{ Labor and delivery ( $n=123$ for intervention group; $n=117$ for comparison group) } \\
\hline Rapid initial assessment & 12 & 60.6 & 0.05 & 42.8 & 0.05 & +17.8 & 0.019 \\
\hline Care during labor & 10 & 81.1 & 0.03 & 66.0 & 0.03 & +15.9 & 0.001 \\
\hline $\begin{array}{l}\text { Assisting women to have safe and clean } \\
\text { birth }\end{array}$ & 13 & 58.9 & 0.04 & 43.7 & 0.04 & +15.2 & 0.003 \\
\hline Immediate newborn care & 4 & 76.9 & 0.04 & 61.9 & 0.04 & +15.0 & 0.013 \\
\hline Preparation of supplies and equipment & 13 & 81.7 & 0.03 & 67.8 & 0.03 & +13.9 & 0.001 \\
\hline Physical and vaginal exam & 18 & 78.1 & 0.04 & 65.2 & 0.04 & +12.9 & 0.013 \\
\hline AMTSL $L^{b}$ & 7 & 82.9 & 0.03 & 71.7 & 0.03 & +11.2 & 0.005 \\
\hline Obstetrical history taking & 8 & 84.9 & 0.06 & 74.6 & 0.06 & +10.3 & 0.240 \\
\hline Quality utilization of partograph & 14 & 77.1 & 0.04 & 70.7 & 0.04 & +6.4 & 0.256 \\
\hline Infection prevention steps & 6 & 92.6 & 0.02 & 87.1 & 0.02 & +5.5 & 0.104 \\
\hline Overall (composite) score & 105 & 77.5 & 0.03 & 65.6 & 0.03 & +11.9 & 0.002 \\
\hline \multicolumn{8}{|c|}{ Immediate postnatal care ( $n=119$ for intervention group; $n=110$ for comparison group) } \\
\hline $\begin{array}{l}\text { Care for the newborn within first hour after } \\
\text { birth }\end{array}$ & 5 & 79.3 & 0.04 & 57.0 & 0.04 & +22.3 & 0.001 \\
\hline Check mother's health after birth & 4 & 66.2 & 0.06 & 44.2 & 0.06 & +22.0 & 0.012 \\
\hline Overall (Composite) score & 9 & 72.8 & 0.05 & 50.6 & 0.03 & +22.2 & 0.001 \\
\hline
\end{tabular}

Results after adjusting for clustering effects

${ }^{\mathrm{b}}$ AMTSL Active management of third stage of labor

\section{Immediate PNC services}

Average overall performance scores for immediate PNC services were significantly higher at intervention than at comparison facilities $(72.8 \%$ versus $50.6 \%, p=$ $0.001)$. There was a significant difference of 22 percentage points between study groups in both skill areas: care for the newborn and health check for the mother (Table 3).

\section{Discussion}

This evaluation found that the SBM-R quality improvement intervention in Ethiopia had a positive impact on $\mathrm{MNH}$ providers' performance in two out of three service areas: uncomplicated labor and delivery care and immediate PNC. Notably, significant and positive impacts on certain important skills that can save the lives of mothers and newborns were found. Rapid initial assessment determines whether pregnant women need emergency care, while immediate newborn care can identify and address potentially life-threatening conditions for newborns. Similarly, active management of the third stage of labor is a proven obstetric intervention for the prevention and management of postpartum hemorrhage [19]. SBM-R also contributed to substantial improvements on postnatal health checks for mother and baby after delivery, which are important to avert key causes of maternal and newborn deaths such as asphyxia, bleeding and severe infection [20]. 
Several factors likely contributed to these improvements. First is BEmONC training conducted in response to initial results of the SBM-R facility assessments, which primarily targeted midwives working in labor and delivery rooms. Training emphasized clinical competencies of labor and delivery care and immediate PNC services and utilized both simulated and clinical settings. Second is the low percentage of institutional deliveries in Ethiopia at the time of the study [21]; the lighter workload may have allowed midwives to take time needed to properly follow SBM-R standards before, during and after delivery. Third, the SBM- $\mathrm{R}$ intervention facilitated mobilization of resources within and outside facilities to address performance gaps. Also, intervention facilities received medical equipment and supplies to improve quality of intra- and postpartum care; this likely increased providers' confidence in their clinical skills.

Findings on labor and delivery care and PNC are generally consistent with evidence from other SBM-R quality improvement interventions directed at $\mathrm{MNH}$ services. For example, studies in Malawi and Tanzania showed significant improvements in PNC performance, such as essential newborn care, at sites that implemented SBM-R $[8,22,23]$. Assessments conducted in 11 low-resource countries in Africa and Latin America found evidence that SBM-R interventions not only caused substantial improvement in labor and delivery service delivery practices such as partograph use and active management of the third stage of labor, but also contributed to positive intermediate and longterm health outcomes, such as lower rates of episiotomy and postpartum hemorrhage and reductions in maternal complications and maternal, neonatal and under five mortality [8, 24-27]. However, systematic evaluations of SBM- $R$ in Malawi and Afghanistan found no significant differences in providers' performance in labor and delivery care between intervention and comparison facilities $[8,22]$.

In contrast to labor and delivery care and PNC, the intervention did not show a significant improvement in providers' performance on ANC. Anecdotal evidence suggests that the lesser impact of the intervention on the quality of ANC performance could be related to providers' qualifications. Unpublished supervision reports from the SBM-R roll-out in Ethiopia indicate that routine ANC is often provided by nurses because the number of midwives at public health facilities is limited. These nurses were trained on general nursing during their pre-service education and did not specialize in $\mathrm{MNH}$. They also did not have the opportunity to receive BEmONC training informed by SBM-R standards. In addition, ANC was often provided to multiple women at the same time, which may have prevented nurses and midwives from meeting SBM- $R$ standards. It is important moving forward to provide refresher training on ANC tasks to all providers who offer these services, including nurses.

Previous studies of the impact of SBM-R interventions on ANC performance have shown mixed results. For example, after SBM-R was introduced at Zambian Defence Force facilities, overall ANC performance increased at intervention sites compared to control sites, but the difference was not statistically significant [28]. Exactly the same pattern was shown in this study, suggesting that SBM-R intervention likely contributed to the wellbeing of maternal, fetal and neonatal health. Other evaluations found that SBM- $\mathrm{R}$ interventions improved providers' performance in ANC services in Afghanistan, but not in Zambia and Malawi $[8,22]$. Another study in Kenya and Afghanistan indicated that quality improvement interventions enhanced ANC services $[19,25]$.

As noted above, many of the practices targeted by this intervention have the potential to save lives. Findings from systematic reviews in low- and middle-income countries confirm that quality improvement interventions focusing on healthcare providers' skills can have a direct impact on health outcomes [8]. For example, SBM-R interventions in West African countries have contributed to decreases in institutional rates of postpartum hemorrhage and maternal and neonatal deaths $[27,29]$. This suggests that scaling up SBM-R could contribute to achieving national goals for the reduction of maternal and newborn mortality rates. This quality model could also contribute to achieving the Ethiopia MOH's 2020 strategic aims to improve intermediate and longterm health outcomes, such as increasing coverage of four ANC visits from 68 to $95 \%$, births attended by skilled health personnel from 60 to $90 \%$, and postnatal coverage from 90 to $95 \%$ [2].

With regard to sustainability, officials who offered supportive supervision and monitored the SBM-R intervention helped in building local ownership of quality improvement strategies at the district and facility levels. Results of the analysis of ICC values suggest that SBM-R contributed to standardization of provider skills in delivering ANC, labor and delivery care and immediate PNC services within and among facilities. Additional activities, such as ongoing mentorship at each facility, may be needed to reinforce and sustain these gains in providers' MNH skills and performance over time.

\section{Strengths and limitations}

Strengths of the study included its reliance on direct observations of routine services by experienced assessors using comprehensive and verifiable tools to assess quality of care by comparing intervention and comparison groups. The biggest limitation is the lack of baseline 
information on provider performance, which makes it impossible to compare changes in provider performance over time between study groups. In future, pre-post study designs are needed to examine the full effects of SBM-R quality improvement tools. In addition, qualitative interview data that could provide a deeper understanding of providers' performance and clients' perspective on the quality of services are lacking. Findings cannot be generalized to all facilities across all regions, and it is possible that other quality improvement approaches fielded by international non-governmental organizations may have contaminated effects in comparison sites. Finally, the study may have introduced the Hawthorne effect, in which providers perform better than usual in response to being observed by external assessors; however, this would have affected both study groups equally.

\section{Conclusions}

The SBM-R quality improvement intervention led to significant improvements in health providers' performance in delivering uncomplicated labor and delivery care and immediate PNC services in Ethiopia, a country with limited resources and a low density of healthcare providers. This substantial change contributed to increased availability of good quality $\mathrm{MNH}$ services at intervention hospitals and health centers. It also strengthened key skills that have the potential to save lives and reduce maternal and newborn mortality.

The Ministry of Health integrated the SBM-R approach within the existing $\mathrm{MNH}$ performance and quality improvement tool; and suggests to scale up across the remaining health facilities in all regions of the country, in order to improve healthcare providers' performance and the quality of care and to contribute to health system strengthening across Ethiopia. These findings will serve as input for implementation of the government's five-year (2015-2020) health sector transformation plan and health care quality strategies to meet the country's sustainable development goals.

\section{Additional file}

Additional file 1: Consent form. (DOCX $40 \mathrm{~kb}$ )

\begin{abstract}
Abbreviations
AMTSL: Active management of third stage of labor; ANC: Antenatal care BEmONC: Basic emergency obstetric and newborn care; ICC: Intraclass correlation coefficient; $\mathrm{MNH}$ : Maternal and newborn health; $\mathrm{MOH}$ : Ministry of Health; PNC: Postnatal care; SBM-R: Standards-Based Management and Recognition; SNNP: Southern Nations, Nationalities and Peoples; USAID: United States Agency for International Development; WHO: World Health Organization
\end{abstract}

\section{Acknowledgements}

We acknowledge the Federal Ministry of Health of Ethiopia for giving permission to conduct the study and the United States Agency for International Development (USAID) for providing financial support. We are also grateful to Professor Judith T. Fullerton and Adrienne Kols for their critical reviews of the manuscript and Dr. Diwakar Mohan for providing guidance in data analysis. Finally, we would like to acknowledge the study participants, the data collectors and supervisors.

\section{Funding}

This study was made possible by the generous support of USAID under the terms of the Leader with Associates Cooperative Agreement GHS-A-00-08-00002-00 of the Maternal and Child Health Integrated Program (MCHIP). The contents are the responsibility of Jhpiego and do not necessarily reflect the views of USAID or the United States Government. USAID did not have any additional role in the study design, data collection and analysis, interpretation of the data, or preparation of the manuscript. Study participants did not receive financial incentives for their participation in the study.

\section{Availability of data and materials}

The datasets used and/or analyzed during the current study are available from the corresponding author on reasonable request.

\section{Authors' contributions}

FA contributed to the study design, managing data collection, performed the data analysis, interpretation of data and wrote the manuscript. NS, JVR, JS and HG contributed to interpretation of data, drafting and reviewing the manuscript. GE, EB and YMK contributed to the development of the study design and writing the manuscript. AT and ED contributed to the study design, participated in data collection, and writing the manuscript. All authors read and agreed to be accountable for the whole process of the manuscript development; and they approved the final manuscript.

\section{Authors' information}

FA: MSc, MA, PhD fellow, Research Team Lead, Jhpiego Ethiopia. GE: MPH, Maternal and Newborn Health Advisor, Jhpiego Ethiopia. NS: MD, MPH, Quality Improvement Advisor, Jhpiego Ethiopia. JVR: Professor, Athena Institute, VU University Amsterdam, the Netherlands. EB: DrPH, MPH, Research Team Lead, Jhpiego USA. YMK: EdD, Senior Research and Evaluation Associate, Jhpiego USA. AT: MPH, Technical Director, Jhpiego Ethiopia. HG: MIH, Registered Midwife, Jhpiego Ethiopia Country Director. ED: MA, Monitoring and Evaluation Team Lead, Jhpiego Ethiopia. JS: Professor, Department of Obstetrics and Gynecology, Leeuwarden Medical Centre and Department of Health Sciences, Global Health, University Medical Centre Groningen, University of Groningen, the Netherlands

\section{Competing interests}

The authors declare that they have no competing interests.

Consent for publication

Not applicable.

Ethics approval and consent to participate

The study was approved by the Johns Hopkins Bloomberg School of Public Health Institutional Review Board and the National Research Ethics Review Committee (NRERC) at the Ethiopian Ministry of Science and Technology to conduct the study in all hospitals and health centers. The NRERC is the highest level of IRB body in Ethiopia to provide ethical approval for all types of researches. In addition, the Ministry of health provided support letter to access selected hospitals and health centers. Written consent was obtained from each study participant. Data were de-identified to maintain confidentiality. See Additional file 1 for the complete consent form for participation.

\section{Publisher's Note}

Springer Nature remains neutral with regard to jurisdictional claims in published maps and institutional affiliations.

\section{Author details}

1Jhpiego Ethiopia, Addis Ababa, Ethiopia. ${ }^{2}$ Athena Institute, VU University, Amsterdam, The Netherlands. ${ }^{3}$ Jhpiego, 1615 Thames St \# 200, Baltimore, MD 21231, USA. ${ }^{4}$ Department of Health Sciences, Global Health, University Medical Centre Groningen, University of Groningen, Groningen, The Netherlands. ${ }^{5}$ Department of Obstetrics and Gynecology, Leeuwarden Medical Centre, Leeuwarden, The Netherlands. 
Received: 26 November 2016 Accepted: 7 April 2017

\section{Published online: 12 April 2017}

\section{References}

1. Countdown to 2015. A decade of tracking progress for maternal, newborn and child survival. The 2015 report. Geneva: UNICEF and World Health Organization; 2015. http://countdown2030.org/documents/2015Report/ CDReport_2015_ex-profiles_final.pdf. Accessed 24 May 2016.

2. Federal Democratic Republic of Ethiopia Ministry of Health. Health Sector Transformation Plan: 2015/16-2019/20. Addis Ababa: Ethiopia Ministry of Health; 2015. http://www.moh.gov.et/documents/26765/0/Health+Sector +Transformation+Plan/5542a23a-9bc7-46a2-8c1f-8b32c2603208?version=1.0. Accessed 24 May 2016

3. World Health Organization (WHO). Standards for improving quality of maternal and newborn care in health facilities. Geneva: WHO; 2016. http://apps.who.int/iris/bitstream/10665/249155/1/9789241511216-eng. pdf. Accessed 20 August, 2016.

4. Rowe AK, de Savigny D, Lanata CF, Victora CG. How can we achieve and maintain high-quality performance of health workers in low-resource settings? Lancet. 2005;366:1026-35.

5. Dettrick Z, Firth $S$, Jimenez Soto E. Do strategies to improve quality of maternal and child health care in lower and middle income countries lead to improved outcomes? A review of the evidence. PLoS One. 2013;8(12), e83070. doi:10.1371/journal.pone.0083070.

6. Tawfik Y, Segall M, Necochea E, Jacobs T. Finding common ground: harmonizing the application of different quality improvement models in maternal, newborn, and child health programs. Bethesda: University Research Co., LLC (URC); 2010. http://www.coregroup.org/storage/ documents/ME/Finding_common_ground.pdf. Accessed 24 May 2016.

7. Althabe F, Bergel E, Cafferata ML, Gibbons L, Ciapponi A, Alemán A, et al. Strategies for improving the quality of health care in maternal and child health in low- and middle-income countries: an overview of systematic reviews. Paediatr Perinat Epidemiol. 2008;22 Suppl 1:42-60.

8. Necochea E, Tripathi V, Kim YM, Akram N, Hyjazi Y, da Luz Vaz M, et al. Implementation of the Standards-Based Management and Recognition approach to quality improvement in maternal, newborn, and child health programs in low-resource countries. Int J Gynaecol Obstet. 2015;130 Suppl 2:S17-24.

9. Lassi ZS, Musavi NB, Maliqi B, Mansoor N, de Francisco A, Toure K, Bhutta ZA. Systematic review on human resources for health interventions to improve maternal health outcomes: evidence from low- and middleincome countries. Human Resour Health. 2016;14:10.

10. Federal Democratic Republic of Ethiopia, Ministry of Health. Basic emergency obstetric \& newborn care (BEmONC) training manual. Addis Ababa: Ethiopia Ministry of Health; 2013. http://pdf.usaid.gov/pdf_docs/ PA00JX4F.pdf. Accessed 7 Nov 2016.

11. Adams G, Gulliford MC, Ukoumunne OC, Eldridge S, Chinn S, Campbell MJ. Patterns of intracluster correlation from primary care research to inform study design and analysis. J Clin Epidemiol. 2004;57:785-94.

12. Eldridge SM, Ukoumunne OC, Carlin JB. The intra-cluster correlation coefficient in cluster randomized trials: a review of definitions. Int Stat Rev. 2009;77:378-94.

13. Getachew A, Ricca J, Cantor D, Rawlins B, Rosen H, Tekleberhan A, et al. Quality of care for prevention and management of common maternal and newborn complications: a study of Ethiopia's hospitals. Baltimore: Jhpiego; 2011. http://www.healthynewbornnetwork.org/hnn-content/uploads/ Ethiopia_QoC_formatted_final.pdf. Accessed 24 May 2016.

14. Scheffler RM, Bruckner T, Spetz J. The labor market for human resources for health in low and middle income countries. Human Resources for Health Observer No. 11. Geneva: World Health Organization; 2012. http://www.who. int/hrh/resources/Observer11_WEB.pdf. Accessed 7 Nov 2016.

15. Park S, Lake ET. Multilevel modeling of a clustered continuous outcome: nurses' work hours and burnout. Nurs Res. 2005;54:406-13.

16. Wears RL. Advanced statistics: statistical methods for analyzing cluster and randomized data. Acad Emerg Med. 2002;9:330-41.

17. Killip S, Mahfoud Z, Pearce K. What is an intracluster correlation coefficient? Crucial concepts for primary care researchers. Ann Fam Med. 2004;2:204-8.

18. Hayes FA. A primer on multilevel modeling. Hum Commun Res. 2006;32:385-410.

19. Tawfik Y, Bongiovanni A, Vaid S, Hermida J, Boucar M, Hill K. Taking every opportunity to save lives: the role of modern quality improvement in enhancing maternal, newborn, and child health programs. A synthesis of USAID Health Care Improvement Project field experience. Health Care Improvement Project Technical Report. Bethesda: University Research Co., LLC (URC); 2012. https://www.usaidassist.org/sites/assist/files/role_of_qi_in_ enhancing_mnch_programs_june2012_0.pdf. Accessed 24 May 2016.

20. Matthews M, Severin VX, Jelka Z. WHO technical consultation on postpartum and postnatal care. Geneva: World Health Organization; 2010. https://www.usaidassist.org/sites/assist/files/role_of_qi_in_enhancing_ mnch_programs_june2012_0.pdf. Accessed 20 Sep 2016.

21. Central Statistical Agency [Ethiopia] and ICF International. Ethiopia Demographic and Health Survey 2011. Addis Ababa: Ethiopia and Calverton, Maryland: Central Statistical Agency and ICF International; 2012.

22. Rawlins BJ, Kim YM, Bazant E, Rashidi T, Bandazi SN, Kachale F, et al. Reproductive health services in Malawi: an evaluation of a quality improvement intervention. Midwifery. 2013:29:53-9.

23. Makene CL, Plotkin M, Currie S, Bishanga D, Ugwi P, Louis H. Improvements in newborn care and newborn resuscitation following a quality improvement program at scale: results from a before and after study in Tanzania. BMC Pregnancy Childbirth. 2014;14:381.

24. Singh K, Brodish P, Speizer I, Barker P, Amenga-Etego I, Dasoberi I, et al. Can a quality improvement project impact maternal and child health outcomes at scale in northern Ghana? Health Res Policy Syst. 2016;14:45.

25. Zainullah P, Kim YM, Bazant E, Yari K. Raising the quality of care: an impact evaluation in Afghanistan. Presented at the ISQua 30th International Conference on Quality and Safety in Population Health and Healthcare. 2013. http://www.isqua.org/docs/default-source/edinburgh/abstract-15minsedinburgh-2013.pdf?sfvrsn=2. Accessed 10 Feb 2016.

26. Kabo M, Otolorin E, Williams E, Orobaton O, Abdullahi H, Sadauki $H$, et al. Monitoring maternal and newborn health outcomes in Bauchi State, Nigeria: an evaluation of a standards-based quality improvement intervention. Int J Qual Health Care. 2016;28:566-72.

27. Rawlins B, Kim YM, Haver J, Rozario A, Kols A, Chiguvare H, et al. Case study: experience applying and tracking a quality improvement approach for maternal and newborn health services in Sub-Saharan Africa. World Health Popul. 2015;16(2):31-8.

28. Kim YM, Chilila M, Shasulwe H, Banda J, Kanjipite W, Sarkar S, et al. Evaluation of a quality improvement intervention to prevent mother-tochild transmission of HIV (PMTCT) at Zambia defence force facilities. BMC Health Serv Res. 2013;13:345

29. Bhutta ZA, Darmstadt GL, Haws RA, Yakoob MY, Lawn JE. Delivering interventions to reduce the global burden of stillbirths: improving service supply and community demand. BMC Pregnancy Childbirth. 2009;9 Suppl 1:S7.

\section{Submit your next manuscript to BioMed Central and we will help you at every step:}

- We accept pre-submission inquiries

- Our selector tool helps you to find the most relevant journal

- We provide round the clock customer support

- Convenient online submission

- Thorough peer review

- Inclusion in PubMed and all major indexing services

- Maximum visibility for your research

Submit your manuscript at www.biomedcentral.com/submit
) Biomed Central 